\title{
Utility of measuring FEV0.75/FVC ratio in preschoolers with uncontrolled wheezing disorder
}

\author{
Véronique Nève ${ }^{1,2,5}$, Sébastien Hulo ${ }^{1,2,5}$, Jean-Louis Edmé ${ }^{1,2}$, Sophie Boileau ${ }^{1}$, \\ Georges Baquet ${ }^{2}$, Guillaume Pouessel ${ }^{3}$, Caroline Thumerelle $^{4}$, \\ Antoine Deschildre ${ }^{4}$ and Régis Matran ${ }^{1,2}$
}

Affiliations: ${ }^{1}$ Pulmonary Function Testing Department, CHU Lille, F59000 Lille, France. ${ }^{2}$ Univ. Lille, Lille, France. ${ }^{3}$ Department of Paediatrics, Hôpital Victor Provo, Roubaix, France. ${ }^{4}$ Department of Paediatric Pneumoallergology, CHU Lille, F59000 Lille, France. ${ }^{5}$ Both authors contributed equally.

Correspondence: Véronique Nève, Pulmonary Function Testing Department, CHU de Lille, 2 avenue Oscar Lambret, 59037 Lille Cedex, France. E-mail: veronique.nevedchru-lille.fr

ABSTRACT Uncontrolled wheezing disorder is common in preschoolers and disease control assessment is challenging as parents frequently overestimate the extent to which their child's disease is controlled. This is the first study of forced expiratory volume in $t \mathrm{~s}(\mathrm{FEV} t)$ /forced vital capacity (FVC) ratio measurements (i.e. FEV1/FVC, FEV0.75/FVC and FEV0.5/FVC) in wheezy preschoolers in relation to disease control. Our objective was to evaluate whether $\mathrm{FEV} t / \mathrm{FVC}$ ratios less than the lower limit of normal (LLN; z-score $<-1.64)$ were associated with uncontrolled wheezing disorder in preschoolers.

Valid FVC, FEV1, FEV0.75 and FEV0.5 values were obtained in 92 healthy and 125 wheezy (62\% uncontrolled) children (3-5 years). Associations between spirometry value $<$ LLN, disease classification (healthy/wheezy) and disease control classifications (controlled/uncontrolled disease) were estimated using logistic regression.

FEV0.75/FVC or FEV0.5/FVC ratios <LLN were associated with wheezing disorder (OR 9.78, 95\% CI 3.70-25.88 and OR 6.64, 95\% CI 2.24-19.66; all p<0.001). Only an FEV0.75/FVC ratio <LLN was associated with uncontrolled wheezing disorder (OR 2.53, 95\% CI 1.12-5.68; $\mathrm{p}=0.025$ ).

FEV $0.75 / F V C$ ratio is a useful surrogate outcome index to evaluate the control of the wheezing disease of preschoolers.

@ERSpublications

FEV0.75/FVC ratio values $<\mathrm{LLN}$ are associated with uncontrolled disease in preschool children with wheezing disorders http://ow.ly/Zl3Sh

This article has supplementary material available from erj.ersjournals.com

Received: Aug 212015 | Accepted after revision: March 082016 | First published online: May 262015

Conflict of interest: None declared.

Copyright OERS 2016 


\section{Introduction}

The assessment of asthma should include assessment of asthma control (both symptom control and future risk of adverse outcomes) [1] and treatment decisions should be based on achieving and maintaining asthma control to obtain significant reductions in the rate of severe exacerbations and improvements in quality of life [2].

In schoolchildren and adults, forced expiratory volume in $1 \mathrm{~s}$ (FEV1) is an important part of the assessment of future risk and serves as an outcome measure for clinical trials. For schoolchildren, the FEV1/forced vital capacity (FVC) ratio appears to be a more sensitive measure of control than FEV1 [3] and the FEV1/FVC ratio was added to the National Heart, Lung, and Blood Institute guidelines as a measure of asthma control [3].

Assessing asthma symptom control in preschool children is important as uncontrolled asthma is common in this group [4]. Healthcare providers are dependent on the report of the child's family members, who might be unaware of the presence of asthma symptoms or the fact that they represent uncontrolled asthma [1]: a recent international survey showed that parents frequently overestimate their child's asthma control [4]. FEV1 may not be an accurate index of bronchial obstruction in preschoolers (as preschoolers often do not exhale for $>1 \mathrm{~s}$ ), and FEV in $0.75 \mathrm{~s}$ or in $0.5 \mathrm{~s}$ (FEV0.75 or FEV0.5) may be more useful outcome measures in this age group [5-7]. Until now, no study has focused on measurements of FEV1/FVC, FEV0.75/FVC and FEV0.5/FVC ratios in preschoolers with wheezing disorders in relation to disease control.

Indices that should always be recorded and reported from spirometry in preschool children are FVC, FEV1, FEV0.75 and FEV0.5 [8]. Reference values for FVC, FEV1 and FEV0.75, but not for FEV 0.5 for preschool children have been provided by the Global Lung Function Initiative (GLI) [9]. It seemed important to obtain reference values for all four indices and references for all three ratios (FEV $/$ FVC, FEV0.75/FVC and FEV0.5/FVC).

Therefore, the aim of the study was to determine, in a preschool population, the most sensitive marker of obstructive lung disease among these three ratios: the first step to reach this objective was to determine reference values for the $\mathrm{FEV} 1 / \mathrm{FVC}, \mathrm{FEV} 0.75 / \mathrm{FVC}$ and $\mathrm{FEV0.5/FVC} \mathrm{ratios} \mathrm{in} \mathrm{healthy} \mathrm{preschool} \mathrm{children} \mathrm{and}$ the second step was to compare these ratios between (uncontrolled/controlled) wheezy and healthy preschool children.

\section{Patients and methods}

Study population

Healthy subjects

Data from 132 healthy Caucasian children, aged 3-5.9 years [10, 11] were collected. The study was designed in accordance with ethical standards of the Helsinki Declaration of 2008 and received approval from the institutional ethics committee (reference 98/23).

\section{Children with wheezing disorders}

Data from 171 children aged 3-5.9 years with recurrent wheezing and asthma diagnosed by a pneumopaediatrician sent to our pulmonary function test laboratory for evaluation were collected [6, 11]. Retrospective review of clinical records provided by the pneumo-paediatrician highlighted the following [12]: wheezing disorder began before the age of 3 years in $68 \%$ of children and persisted after the age of 6 years in 54\%; the presence of atopy was noted in $78 \%$ of children (atopic dermatitis in 35\%, food allergy in $19 \%$ and/or allergic rhinitis in 11\%); sensitisation to aeroallergens (assessed by immediate hypersensitivity skin testing or an in vitro method that detects antigen-specific IgE antibody) was noted in $58 \%$ of children; asthma was partly controlled/uncontrolled in $62 \%$ of children (assessment according to Global Initiative for Asthma (GINA) guidelines [13]). Maintenance treatment administered to the child at the time of the pulmonary function tests included inhaled corticosteroids with a spacer in $92 \%$ of children ( $>400 \mu \mathrm{g}$ daily beclometasone equivalent in $85 \%$ of children). Inhaled corticosteroids were associated with inhaled short-acting $\beta_{2}$-agonists or ipratropium in $6 \%$ of children and inhaled long-acting $\beta_{2}$-agonists in $21 \%$ of children and/or antihistamine medication in $41 \%$ of children. Inhaled $\beta_{2}$-agonists and muscarinic antagonists were withheld for $>12 \mathrm{~h}$ before testing.

\section{Recording of flow-volume curves}

Spirometry was performed using a Masterscreen with Jaeger incentive programmes (Erich Jaeger, Wurzburg, Germany) [6]. Thus, up to 15 manoeuvres could be requested from each child with up to 10 manoeuvres per session being selected by the nurses and recorded. All curves were then visually reviewed for technical acceptability [8] by study staff (V. Nève) blinded to their clinical status. Salbutamol was only administered to wheezy children. Bronchodilator reversibility was assessed 20 min after administration of inhaled salbutamol $(200 \mu \mathrm{g})$ via a spacer device. 
Acceptability and repeatability

Acceptability and repeatability criteria were applied as previously described [6] and followed the American Thoracic Society/European Respiratory Society recommendations (2007) [8]. The start-of-test criteria (the back extrapolated volume and the extrapolated volume/forced vital capacity (FVC) ratio [14]) were not used as exclusion criteria per se [8]. If cessation of flows occurred at $>10 \%$ of peak expiratory flow then this manoeuvre was classified as showing premature termination and timed expiratory volumes (FEV in $t \mathrm{~s}$ $(\mathrm{FEV} t)$, such as FEV1, FEV0.75 and FEV0.5) from such manoeuvres were reported, while unacceptable FVC was not [8]. Repeatability was assessed by calculating absolute and percentage differences $(\Delta)$ between the two best FVC, FEV1, FEV 0.75 and FEV0.5. The largest FVC, the largest FEV1, the largest FEV0.75 and the largest FEV0.5 from any of the technically acceptable manoeuvres were reported [8].

Data were available on 132 healthy and 171 wheezy preschool children (fig. 1). Those with an acceptable FVC (105 healthy and 151 wheezy children) were included in this study. The number of acceptable FEV1 data was smaller than that of FEV0.75, therefore analyses were performed on children with acceptable FVC, FEV1, FEV0.75 and FEV0.5 (92 healthy and 125 wheezy children).

\section{Data analysis}

Reference values were determined as described by Nève et al. [10] and in the online supplementary material. Briefly, data were obtained from 446 Caucasian children (aged 2.5-15 years) [10]. The lambda-mu-sigma method was applied. Data were analysed using the GAMLSS (generalised additive model for location scale and shape) package 4.1-1 in the statistical programme R (R Software 2.14.1; R Development Core Team, 2008, www.r-project.org). Reference equations for FEV $t / \mathrm{FVC}$ ratios are provided in online supplementary table E1 (reference equations for FEV1 and FVC are provided in [10]) and the coefficient of variation for FEV $t / F V C$ in online supplementary figure E1.

Spirometric data obtained in healthy or wheezy children were expressed as z-scores from reference values obtained in our healthy children. Z-score values $<-1.64$ were considered to be below the lower limit of normal (LLN) [15]. All spirometric indices were converted to categorical variables indicating values $<$ LLN ( $\mathrm{z}$-score $<-1.64$ into " 1 ") or value $\geqslant \operatorname{LLN}(\mathrm{z}$-score $\geqslant-1.64$ into " 0 ").

Data were expressed as a percentage for qualitative variables and as mean \pm SD for continuous variables. Differences in proportions and means were analysed using the Pearson Chi-squared test or Fisher exact test and ANOVA. The association between values of spirometry indices <LLN and disease classifications (healthy/wheezy) or disease control classifications (controlled/ uncontrolled disease) was estimated as OR (95\% CI) using logistic regression analyses. For FEV0.75/FVC ratio, we also calculated OR (95\% CI) using resampling bootstrapping, based on 1000 iterations [16]. Logistic regressions and the bootstrap analyses were performed using SAS (version 9.4; SAS Institute, Cary, NC, USA). Repeated-measure ANOVA was used to assess the effect of salbutamol on FEV1 and FEV0.75 measurements.

Valid FVC li.e. without premature termination and with cessation of flow occuring at $<10 \%$ of PEFl was obtained in:

Valid FVC and reportable FEV 0.75 and reportable FEV1 were obtained in:
Data of 171 children with wheezing disorders were collected

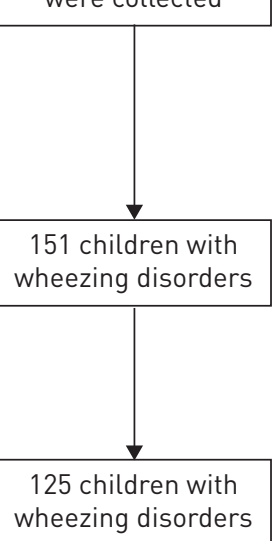

FIGURE 1 Flow chart diagram of recruited and analysed children. FVC: forced vital capacity; PEF: peak expiratory flow; FEV1 and FEV0.75: forced expiratory volume in $1 \mathrm{~s}$ and $0.75 \mathrm{~s}$, respectively. 


\section{Results}

\section{Healthy children}

Among the 132 children aged 3-5 years (fig. 1), at least two acceptable curves [8], where the second highest FVC and FEVt were within $0.1 \mathrm{~L}$ and $10 \%$ of the highest value, were obtained in $95 \%, 92 \%, 94 \%$ and $98 \%$ for FVC, FEV1, FEV0.75 and FEV0.5, respectively, and in 105 children, acceptable FVC was obtained (fig. 1) with reportable FEV1 and FEV0.75 in 92 and 102 children, respectively.

In 92 children acceptable FVC and reportable FEV1, FEV0.75 and FEV0.5 were obtained (fig. 1). Their baseline spirometric measurements, as z-scores from Lille reference equations are shown in table 1. Compared to those of the 13 children without acceptable FVC, these children had comparable anthropometric data (online supplementary table E2).

Among healthy children, 3.3\%, 7.6\%, 1.1\%, 6.5\% and 0\% had spirometric z-score values <-1.64 from GLI 2012 reference equations [9] for FVC, FEV1, FEV0.75, FEV1/FVC and FEV0.75/FVC, respectively.

\section{Children with wheezing disorders}

Among the 171 wheezy children aged 3-5 years (fig. 1), at least two acceptable curves [8], where the second highest FVC and FEV $t$ were within $0.1 \mathrm{~L}$ and $10 \%$ of the highest value, were obtained in $86.3 \%, 88.9 \%, 95.3 \%$ and $91.5 \%$ for FVC, FEV1, FEV0.75 and FEV0.5, respectively and, in 151 children, acceptable FVC was obtained (fig. 1) with reportable FEV1 and FEV0.75 in 129 and 143 of them, respectively.

In 125 children with wheezing disorders, acceptable FVC and reportable FEV1, FEV0.75 and FEV0.5 were obtained (fig. 1). Their mean age was comparable to that of healthy children (table 1), but their height-for-age was lower and they were more likely to be male. Compared to the 26 children without acceptable FVC these children were older but their height-for-age was comparable (online supplementary table E2).

Wheezy children had lower FEV0.75, FEV0.5, FEV0.75/FVC ratio and FEV0.5/FVC ratio values expressed as $\mathrm{z}$-scores (table 1) than healthy children, but FEV1 and the FEV1/FVC ratios were not significantly different between the groups. The same results were obtained on a larger population with different sample sizes (that of fig. 1, second line) for every index (sensitivity analysis in online supplementary table E3).

Percentages of children with spirometric data values $<$ LLN are shown in table 2.

Compared to healthy children, a larger proportion of wheezy children showed FVC z-scores, FEV0.75 z-scores, FEV0.5 z-scores, FEV0.75/FVC ratio z-scores or FEV0.5/FVC ratio z-scores <LLN (table 2), but proportions of wheezy and healthy children with FEV1 z-scores or FEV1/FVC ratio z-scores <LLN did not differ. The same results were observed for $\mathrm{FEV}_{1} / \mathrm{FVC}$ and $\mathrm{FEV} 0.75 / \mathrm{FVC}$ when data were standardised to the GLI reference equations (data not shown).

Figure 2 shows that wheezing disease was associated with higher odds of values <LLN for FVC, FEV0.75, FEV 0.5, FEV0.75/FVC or FEV0.5/FVC ratios, but not for FEV1 and FEV1/FVC ratio.

TABLE 1 Spirometric indices of healthy children and children with wheezing disorder calculated from Lille reference equations

\begin{tabular}{|c|c|c|c|}
\hline & Healthy & Wheezy & $\begin{array}{l}\text { ANOVA } \\
\mathrm{p} \text {-value }\end{array}$ \\
\hline Subjects $n$ & $92^{\#}$ & $125^{\pi}$ & \\
\hline Males & $43(46.7)$ & $84(67.2)$ & 0.003 \\
\hline Age years & $4.53 \pm 0.75$ & $4.58 \pm 0.88$ & 0.629 \\
\hline Height-for-age z-score [17] & $0.52 \pm 0.93$ & $0.18 \pm 1.10$ & 0.013 \\
\hline FVC z-score & $0.03 \pm 0.97$ & $-0.10 \pm 1.32$ & 0.442 \\
\hline FEV 1 z-score & $0.04 \pm 0.96$ & $-0.04 \pm 1.09$ & 0.620 \\
\hline FEV0.75 z-score & $0.01 \pm 0.97$ & $-0.79 \pm 1.20$ & $<10^{-4}$ \\
\hline FEV 0.5 z-score & $-0.02 \pm 1.00$ & $-0.52 \pm 1.20$ & 0.002 \\
\hline FEV1/FVC z-score & $0.09 \pm 0.97$ & $0.04 \pm 1.25$ & 0.719 \\
\hline FEV0.75/FVC z-score & $0.08 \pm 1.00$ & $-1.33 \pm 1.71$ & $<10^{-4}$ \\
\hline FEV0.5/FVC z-score & $-0.04 \pm 0.94$ & $-0.74 \pm 1.40$ & $<10^{-4}$ \\
\hline
\end{tabular}


TABLE 2 Percentages of healthy and wheezy children with spirometric data below the lower limit of normal (i.e. with z-score values according to Lille reference equations <-1.64)

\begin{tabular}{lccc} 
& Healthy & Wheezy & $\begin{array}{c}\text { Chi-squared } \\
\text { p-value }\end{array}$ \\
\hline Subjects n & $92^{\#}$ & $125^{\text {q }}$ & \\
FVC & $4(4.3)$ & $16(12.8)$ & 0.033 \\
FEV & $3(3.3)$ & $10(8.0)$ & 0.146 \\
FEV 0.75 & $5(5.4)$ & $29(23.2)$ & $<0.001$ \\
FEV0.5 & $5(5.4)$ & $24(19.2)$ & 0.003 \\
FEV1/FVC & $4(4.6)$ & $14(11.2)$ & 0.071 \\
FEV0.75/FVC & $45(36.0)$ & $<10^{-4}$ \\
FEV0.5/FVC & $5(5.4)$ & $29(23.2)$ & $10^{-4}$ \\
\hline
\end{tabular}

Data are presented as $\mathrm{n}(\%)$, unless otherwise stated. FVC: forced vital capacity; FEVt: forced expiratory volume in $t \mathrm{~s} .{ }^{\#}: \mathrm{n}=30$ 3-year-old children, $\mathrm{n}=31$ 4-year-old children and $\mathrm{n}=31$ 5-year-old children; ${ }^{\text {ๆ }} \mathrm{n}=38$ 3 -year-old children, $n=38$ 4-year-old children and $n=49$-year-old children.

Spirometry indices recorded after administration of inhaled salbutamol were obtained in 76 wheezy children for FEV1 and 81 wheezy children for FEV0.75. Mean \pm SD FEV1 increased from $1.016 \pm 0.214 \mathrm{~L}$ to $1.115 \pm 0.242 \mathrm{~L}$ $(\mathrm{p}<0.0001)$ and mean \pm SD FEV0.75 increased from $0.883 \pm 0.187 \mathrm{~L}$ to $0.988 \pm 0.212 \mathrm{~L}(\mathrm{p}<0.001)$.

\section{Uncontrolled versus controlled wheezing disorder}

Baseline spirometric measurements, expressed as z-scores, of the 78 children with uncontrolled disease and the 47 with controlled wheezing disorders were not statistically different (online supplementary table E4).

A larger proportion of wheezy children with uncontrolled disease $(43.6 \%)$ than children with controlled disease $(23.4 \%, \mathrm{p}=0.002)$ showed $\mathrm{FEV} 0.75 / \mathrm{FVC}$ ratio $\mathrm{z}$-scores below the expected "normal range", but proportions of children with uncontrolled and controlled disease with FEV1/FVC ratio z-scores <LLN ( $10.3 \%$ versus $12.8 \%, \mathrm{p}=0.666)$ or $\mathrm{FEV} 0.5 / \mathrm{FVC}$ ratio $\mathrm{z}$-scores $<\mathrm{LLN}(28.2 \%$ versus $14.9 \%, \mathrm{p}=0.087)$ did not differ. Figure 3 shows that uncontrolled wheezing disease was associated with higher odds of values $<$ LLN for FEV0.75/FVC (OR 2.46, 95\% CI 1.12-5.42), but not for FVC, FEV1, FEV0.75, FEV0.5, FEV1/FVC ratios or FEV0.5/FVC ratios. The bootstrap method confirmed that uncontrolled wheezing disease was associated with higher odds of values < LLN for FEV0.75/FVC (OR 2.62, 95\% CI 1.14-6.32).

\section{Discussion}

Our study analysed the FEV1/FVC, FEV0.75/FVC and FEV0.5/FVC ratios of wheezy preschool children in order to investigate whether these ratios were associated with uncontrolled wheezing disease. We had first determined reference values for these ratios and the LLN by using $\mathrm{z}$-scores from data obtained in healthy children. Our results suggest that the FEV0.75/FVC ratio is significantly associated with the control of wheezing disease in preschool children, where the FEV0.5/FVC and FEV1/FVC ratios are not.

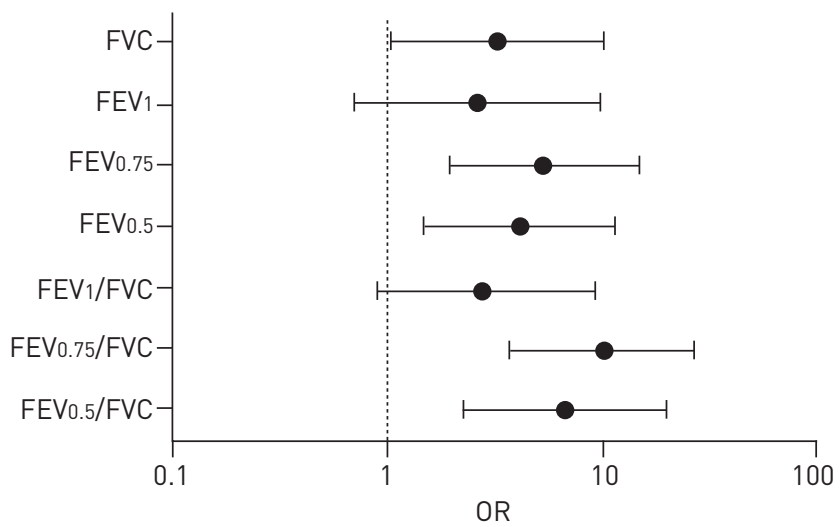

FIGURE 2 Logistic regression analyses for the association with the wheezing condition compared with the healthy condition. It shows that wheezing disease was associated with higher odds of values less than the lower limit of normal (LLN) for forced vital capacity (FVC), forced expiratory volume in $0.75 \mathrm{~s}$ (FEV 0.75 ) or $0.5 \mathrm{~s}$ (FEV 0.5 ), FEV $0.75 / F V C$ or $F E V_{0.5} / F V C$ ratio, but not for $F E V$ in $1 \mathrm{~s}\left(F E V_{1}\right)$ or for $\mathrm{FEV}_{1} / \mathrm{FVC}$ ratio. 


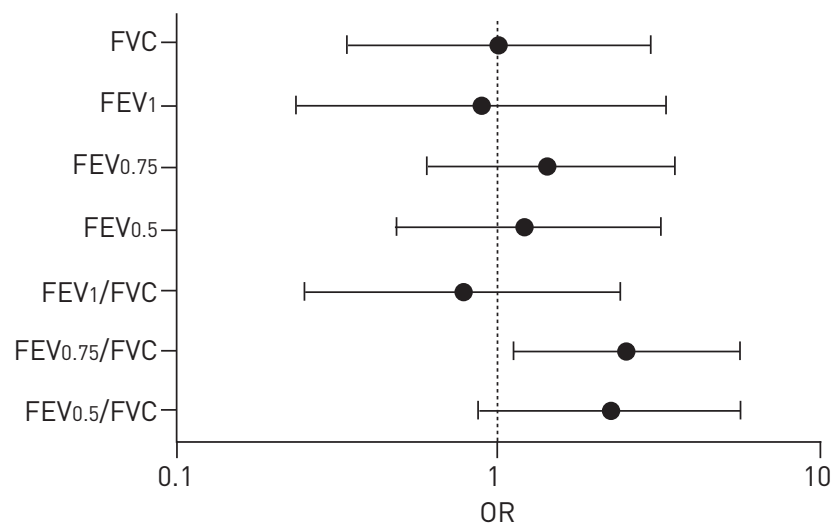

FIGURE 3 Odds ratios for forced vital capacity (FVC), forced expiratory volume in $t \mathrm{~s}$ (FEVt) and FEVt/FVC ratios in uncontrolled versus controlled wheezy children. Uncontrolled wheezing disease was associated with higher odds of values less than the lower limit of normal for FEV0.75/FVC (OR 2.46, 95\% Cl 1.12-5.42), but not for $\mathrm{FVC}, \mathrm{FEV} 1, \mathrm{FEV} 0.75$, FEV0.5, FEV1/FVC ratio or FEV0.5/FVC ratio.

\section{FEV0.75/FVC and FEV0.5/FVC}

This is the first study on FEVt/FVC ratio measurement in wheezy preschoolers in relation to disease control. It is the first to demonstrate that the FEV0.75/FVC ratio is significantly associated with the control of the wheezing disease in preschool children, but that the FEV0.5/FVC ratio is not. Moreover, our results from groups including healthy and wheezy preschool children show that FEV0.75/FVC and FEV0.5/FVC ratio z-score values <LLN are associated with an increased likelihood of having wheeze disorder. Our results confirm that an assessment of FEV0.75 or FEV0.5 is more clinically relevant than FEV 1 in the preschool age group. Indeed, in healthy and wheezy preschool children FEV0.75 and FEV0.5 z-score values $<$ LLN were associated with an increased likelihood of having wheeze disorder. VILOzNI et al. [7] showed that FEV0.5 was sensitive in detecting moderate and severe symptoms (in contrast to FEV1, which was sensitive only to severe symptoms). Diagnosing asthma is challenging in preschool children; FEV0.75, FEV0.5, FEV0.75/FVC ratio and FEV0.5/FVC ratio appear to be useful outcome surrogate indices that are more clinically relevant than $\mathrm{FEV}_{1}$ or $\mathrm{FEV} 1 / \mathrm{FVC}$ ratio for diagnosing wheeze disorder or uncontrolled disease in this age group.

Complete asthma control is uncommon in children [4]: a large international asthma survey conducted with parents of 4-15-year-old asthmatic children showed that $85 \%$ of children had incompletely controlled asthma as defined by GINA guidelines, and that parents overestimated their child's asthma control. As FEV0.75/FVC ratio was the only index associated with uncontrolled disease in our study, it could be a useful surrogate outcome index to provide an assessment of symptom control based on GINA guidelines. The qualitative analysis based on the percentage of children having an FEV0.75/FVC ratio $<-1.64$ z-score seems particularly interesting in this regard, as it showed a higher prevalence of FEV0.75/FVC ratio $<$ LLN in children with uncontrolled than in controlled wheezing disorders.

\section{FEV $1 / F V C$}

Our results show that the FEV1/FVC ratio is not significantly associated with the control of wheezing disease in preschool children and that it fails to confirm the diagnosis of wheeze disorder in populations of healthy and wheezy preschool children. In preschool children with wheeze disorder, studies of the utility of the FEV1/FVC ratio are scarce and none have specifically evaluated the association of the FEV1/ FVC ratio with uncontrolled disease. GAFFin et al. [18] also observed in a population of preschool children that included children with wheeze disorder that this index was close to $100 \%$ predicted and that as few as $14 \%$ of $\mathrm{FEV} 1 / \mathrm{FVC}$ ratios were $<85 \%$ pred. In addition, while in schoolchildren the $\mathrm{FEV} 1 / \mathrm{FVC}$ ratio measurement decreased with increasing asthma severity, on the basis of symptom frequency [19, 20], in wheezy preschool children, the FEV1/FVC ratio was shown to be insensitive to disease severity (as evaluated according to GINA guidelines) [7] and mean FEV1/FVC ratio remained in the normal range while disease severity increased. The FEV1/FVC ratio may be less relevant in this age group because most preschool children can barely achieve forced expiration lasting $1 \mathrm{~s}$ and the FEV1/FVC ratio for most children may be close to 1.00. Finally, the decrease in FEV1/FVC ratio with age that was demonstrated in the preschool- and school-aged children $[9,21]$ must be taken into account. Our results have accounted for the age change in predicted value and the age change in intersubject variability.

Our results show that FEV1 $\mathrm{z}$-score <LLN fails to detect either the uncontrolled wheezing disease or wheeze disorder in a preschool population. These results are in agreement with cross-sectional studies that 
found normal FEV1 values in a majority of the school-aged [19, 20, 22] and preschool [18] children with asthma. In the latter study, few children with wheeze disorder had an FEV1 $<80 \%$ pred. ViLOzNi et al. [7] added that the FEV1 was sensitive in detecting only severe symptoms. As healthy children have higher elastic recoil than healthy adults, with faster emptying of the lung, the FEV1 may be relatively insensitive as an indication of early-stage lung disease $[10,23]$.

\section{Physiological reasons that could explain why FEV0.75 is more sensitive than FEV1 in this age group}

In older children and adults, the physiological and clinical utility of FEV1 is due to its location on the effort-independent (flow-limited) part of the maximum expiratory flow-volume loop, which descends to lung volumes as low as $85-90 \%$ of exhaled vital capacity and reflects the intrinsic mechanical properties of the respiratory system. The ability to maintain flow limitation at low lung volumes depends largely on the strength of the chest wall muscles to maintain sufficient driving pressure. It is unlikely that children in the preschool age group possess sufficient expiratory muscle strength to maintain flow limitation to lung volumes as low as $90 \%$ of exhaled vital capacity [24]. The final effort-dependent portion of the FEV1, unlike FEV0.75 and FEV0.5, can obscure flow limitation by adding "noise" to the signal.

\section{Strengths and weaknesses of this study}

Strengths of our study include that spirometry was performed by the same skilled nurses and with the same device for healthy and wheezy children, limiting technical sources of variability, and that reference values were derived from data obtained from children of the same ethnicity and living in the same region of northern France. We acknowledge that our sample size in the 3-5-year age range was limited and the 95\% confidence intervals of the odds ratios are rather large but, but our findings were confirmed in a sensitivity analysis carried out in a larger population of healthy/wheezy children. In addition, to confirm the results obtained by logistic regression, a robustness analysis using resampling bootstrap was performed: the bootstrap was suitable because it was based on very limited assumptions about the probability distribution that gave rise to the data [16]. The bootstrapping confirmed that uncontroled wheezing diseases was associated with higher odds of values $<$ LLN for the FEV0.75/FVC ratio.

In conclusion, the FEV0.75/FVC ratio appears to be a useful surrogate outcome parameter index to evaluate the control of the wheezing disease of preschool children, while FEV0.5/FVC and FEV1/FVC ratios are not associated with disease control. Early intervention is crucial in treating asthma because it can improve symptom control, reduce frequent exacerbations and retard the progression of airway remodelling. When wheeze disorder develops before the third birthday, significant lung damage can occur by the age of 6 years [25]. FEV0.75 and FEV0.75/FVC ratio appear to be particularly suited to the evaluation of airway function in preschoolers with wheezing disorders.

\section{Acknowledgements}

The authors are greatly indebted to Catherine-Marie Methlin and Christelle Delille (Pulmonary Function Testing Department, CHU Lille, Lille, France) for performing the pulmonary function tests. They thank all the children from the schools of Illies, Herlies and La Bassée (France), who participated enthusiastically in this study. They also thank the Inspection d'Académie of Lille (France), and are grateful to Mike Howsam (Univ. Lille, Lille, France) for help with the translation of the manuscript.

\section{References}

1 NHLBI/WHO Report. Global Initiative for Asthma (GINA). Global Strategy for Asthma Management and Prevention. NIH Publication No 02-3659. Issued January, 1995 (updated 2012).

2 Bateman ED, Boushey HA, Bousquet J, et al. Can guideline-defined asthma control be achieved? The Gaining Optimal Asthma ControL study. Am J Respir Crit Care Med 2004; 170: 836-844.

3 National Asthma Education and Prevention Program. Expert panel report 3 (EPR-3): guidelines for the diagnosis and management of asthma - summary report 2007. J Allergy Clin Immunol 2007; 120: Suppl. 5, S94-S138.

4 Carroll WD, Wildhaber J, Brand PL. Parent misperception of control in childhood/adolescent asthma: the Room to Breathe survey. Eur Respir J 2012; 39: 90-96.

5 Aurora P, Stocks J, Oliver C, et al. Quality control for spirometry in preschool children with and without lung disease. Am J Respir Crit Care Med 2004; 169: 1152-1159.

6 Nève V, Edmé JL, Devos P, et al. Spirometry in 3-5-year-old children with asthma. Pediatr Pulmonol 2006; 41: 735-743.

7 Vilozni D, Barak A, Efrati $\mathrm{O}$, et al. The role of computer games in measuring spirometry in healthy and "asthmatic" preschool children. Chest 2005; 128: 1146-1155.

8 Beydon N, Davis SD, Lombardi E, et al. An official American Thoracic Society/European Respiratory Society statement: pulmonary function testing in preschool children. Am J Respir Crit Care Med 2007; 175: 1304-1345.

9 Quanjer PH, Stanojevic S, Cole TJ, et al. Multi-ethnic reference values for spirometry for the 3-95-yr age range: the global lung function 2012 equations. Eur Respir J 2012; 40: 1324-1343.

10 Nève V, Edmé JL, Baquet G, et al. Reference ranges for shape indices of the flow-volume loop of healthy children. Pediatr Pulmonol 2015; 50: 1017-1024.

11 Nève V, Matran R, Baquet G, et al. Quantification of shape of flow-volume loop of healthy preschool children and preschool children with wheezing disorders. Pediatr Pulmonol 2012; 47: 884-894. 
12 Reddel HK, Taylor DR, Bateman ED, et al. An official American Thoracic Society/European Respiratory Society statement: asthma control and exacerbations: standardizing endpoints for clinical asthma trials and clinical practice. Am J Respir Crit Care Med 2009; 180: 59-99.

13 National Heart, Lung, and Blood Institute (NHLBI), World Health Organization (WHO). NHLBI/WHO Workshop Report. Global Initiative for Asthma (GINA). Global Strategy for Asthma Management and Prevention. Bethesda, MD, National Institutes of Health, 2002 (updated 2009).

14 Nève V, Matran R, Edmé JL. Quality control for spirometry in preschool children. Am J Respir Crit Care Med 2008; 178: 107.

15 Pellegrino R, Viegi G, Brusasco V, et al. Interpretative strategies for lung function tests. Eur Respir J 2005; 26 : 948-968.

16 Quintana HK, Vikström M, Andersson T, et al. Agreement between myocardial infarction patients and their spouses on reporting of data on 82 cardiovascular risk exposures. PLoS One 2015; 10: e0132601.

17 Cole TJ, Freeman JV, Preece MA, et al. British 1990 growth reference centiles for weight, height, body mass index and head circumference fitted by maximum penalized likelihood. Stat Med 1998; 17: 407-429.

18 Gaffin JM, Shotola NL, Martin TR, et al. Clinically useful spirometry in preschool-aged children: evaluation of the 2007 American Thoracic Society Guidelines. J Asthma 2010; 47: 762-767.

19 Bacharier LB, Strunk RC, Mauger D, et al. Classifying asthma severity in children: mismatch between symptoms, medication use, and lung function. Am J Respir Crit Care Med 2004; 170: 426-432.

20 Paull K, Covar R, Jain N, et al. Do NHLBI lung function criteria apply to children? A cross-sectional evaluation of childhood asthma at National Jewish Medical and Research Center, 1999-2002. Pediatr Pulmonol 2005; 39: 311-317.

21 Eigen H, Bieler H, Grant D, et al. Spirometric pulmonary function in healthy preschool children. Am J Respir Crit Care Med 2001; 163: 619-623.

22 Spahn JD, Cherniack R, Paull K, et al. Is forced expiratory volume in one second the best measure of severity in childhood asthma? Am J Respir Crit Care Med 2004; 169: 784-786.

23 Mead J, Turner JM, Macklem PT, et al. Significance of the relationship between lung recoil and maximum expiratory flow. J Appl Physiol 1967; 22: 95-108.

24 Joseph-Bowen J, de Klerk NH, Firth MJ, et al. Lung function, bronchial responsiveness, and asthma in a community cohort of 6-year-old children. Am J Respir Crit Care Med 2004; 169: 850-854.

25 Martinez FD, Wright AL, Taussig LM, et al. Asthma and wheezing in the first six years of life. N Engl J Med 1995; 332: $133-138$. 